\title{
Moisés Poblete Troncoso y la primera Historia del movimiento obrero latinoamericano (1946)
}

\author{
Moisés Poblete Troncoso and the first History of the Latin-American \\ Labor Movement (1946)
}

\author{
Juan Carlos Yáñez Andrade*
}

Resumen: El libro El movimiento obrero latinoamericano del intelectual y abogado chileno Moisés Poblete Troncoso puede ser considerado la primera investigación histórica que buscó abordar de manera conjunta la evolución del sindicalismo continental. Publicado en 1946 por el Fondo de Cultura Económica, el año 2016 se cumplieron 70 años de su aparición. El objetivo del presente artículo es conocer el contexto de su publicación y las razones que motivaron a Poblete a dar forma a una obra pionera, la que marcó los estudios sobre el sindicalismo latinoamericano.

Palabras clave: Moisés Poblete, movimiento obrero latinoamericano, sindicalismo, OIT.

\begin{abstract}
The book The Latin-American Labor Movement of the intellectual and lawyer Chilean Moisés Poblete Troncoso can be considered the first historical research that seeks to jointly address the evolution of continental trade unionism. Published in 1946 by the Fondo de Cultura Económica, the year 2016 marked the 70th anniversary of its appearance. The objective of this article is to know the context of its publication and the reasons that motivated Poblete to give shape to a pioneering work, which marked the studies on Latin-American trade unionism.
\end{abstract}

Keywords: Moisés Poblete, Latin-American Labor Movement, Trade-unions, ILO

Recibido: $26 / 04 / 2017$

Aceptado: 25/06/2017

\footnotetext{
* Doctor en Historia por la EHESS-París. Académico de la Universidad de Valparaíso. Correo electrónico: juancarlos.yanez@uv.cl. Una versión preliminar de este artículo fue presentada al V Congreso Latinoamericano de Historia Económica (CLADHE), desarrollado en Sao Paulo entre el 19 y 21 de julio del 2016. Agradezco a los asistentes por sus valiosos comentarios.
} 


\section{Introducción}

En los últimos años los estudios transnacionales han tenido un desarrollo inesperado, ofreciendo miradas renovadoras sobre los procesos regionales, las instituciones internacionales y los actores que actúan en el dominio supranacional. Si bien las unidades espaciales y políticas como la Nación o el Estado siguen siendo pertinentes para conocer aspectos importantes de la evolución de lo social, los estudios trasnacionales se caracterizan por vincular estas unidades con áreas de circulación regional y conexiones globales ${ }^{1}$. El campo social supone un dominio donde diferentes actores actúan y buscan legitimarse frente a problemas que no son ni políticos, ni económicos estrictamente hablando, sino sociales. Es decir, aquellos que conciernen un amplio espectro que puede ir de los problemas del trabajo a las condiciones de vida. Frente a una historiografía latinoamericana centrada en los procesos de formación del Estado Nación o en la dimensión nacional de las temáticas que se analizan, las perspectivas transnacionales de lo social se sitúan en el cruce de la historia del trabajo, de las instituciones internacionales, de la cooperación regional, de las políticas sociales y de la historia de la producción de saberes.

El papel de las organizaciones internacionales que se crearon al alero del internacionalismo ginebrino luego de la Primera Guerra Mundial son un buen punto de apoyo para pensar la formación contemporánea de América Latina -parafraseando a Akira Iriye $^{2}$-, ya que ayudaron a movilizar los esfuerzos en pro de pensar regionalmente los problemas sociales. En cierta forma, instituciones internacionales como la Organización Internacional del Trabajo (OIT), aparecen como representativas de una nueva forma de articular intereses en pugna, tanto gubernamentales, empresariales, como sindicales, en un contexto marcado por la necesidad de consolidar la paz y garantizar el progreso económico a una amplia mayoría. A este panel de instituciones internacionales que comienzan a operar en el continente americano, podemos agregar las oficinas del trabajo, los museos sociales (especialmente el Museo Social Argentino), las organizaciones filantrópicas, como la Dotación Carnegie o la Fundación Rockefeller, y algunas federaciones sindicales.

Una ventaja de la historia transnacional con respecto a las perspectivas tradicionales, es que permite multiplicar el recorrido de los actores que participan en la configuración del espacio social continental y, además, ayuda a clarificar las instancias de convergencia que se dan en cada proceso histórico, como es en nuestro caso la formación de lo socio-laboral. Es a partir de esta noción de convergencia que se puede entender la creación de ciertos consensos entre los países en el respeto a ciertas normas internacionales del trabajo o en el reconocimiento a ser parte de una comunidad de valores, y que explica, en último término, la creación de acuerdos internacionales, la participación en conferencias continentales y la

\footnotetext{
${ }^{1}$ Para una aproximación general, pero que ofrece múltiples entradas a los estudios transnacionales, se puede consultar la publicación coordinada por Pierre-Yves Saunier y Akira Iriye, The Palgrave Dictionary of Transnational History, New York, MacMillan Publisher, 2009.

${ }^{2}$ Akira Iriye, Global Community. The Role of International Organizations in the Making of the Contemporary World, Berkeley, California University Press, 2002.
} 
integración a redes transnacionales.

Sin este contexto transnacional favorable, sería difícil poder comprender el recorrido de un intelectual como Moisés Poblete Troncoso, abogado chileno que fue parte del debate socio-laboral chileno durante la década de 1920 y que se transformó en funcionario internacional a fines de esa década.

El objetivo central de este artículo es reconstruir la evolución intelectual que tuvo Poblete durante los años 1920, 1930 y 1940, a través de un análisis de su obra y sus vinculaciones tanto nacionales como internacionales, destacando su aporte al conocimiento de la legislación social y laboral de Chile y del continente. Aspecto esencial, y que justifica este artículo, es analizar su libro El movimiento obrero latinoamericano, publicado en México en 1946 por el Fondo de Cultura Económica. Nos interesa, en particular, vincular esta obra con el dominio de preocupaciones que tenía Poblete desde los años 1920 sobre la condición laboral de los trabajadores, destacando cómo su paso por la OIT entre 1927 y 1937 fortaleció sus competencias y amplió sus vinculaciones con intelectuales, dirigentes sindicales y autoridades de gobierno, lo que sería clave en el esfuerzo recopilatorio que significó El movimiento obrero latinoamericano.

En términos formales, el artículo se organiza en tres secciones, que comprenden, primero, una presentación de los primeros estudios que Poblete llevó a cabo desde que egresó como abogado, hasta sus publicaciones cuando fue director de la Oficina del Trabajo entre 1920 y 1925. A continuación, se describen algunos procesos político-sindicales que se dieron en el continente desde comienzos del siglo XX, hasta las condiciones favorables de la década de 1930 para unificar a los trabajadores del continente. Sus vínculos con personalidades claves como el dirigente sindical mexicano Vicente Lombardo Toledano o el reconocimiento que comienza a tener la OIT en el continente, ayudan a entender el contexto proclive a la recepción de El movimiento obrero latinoamericano. Por último, en una tercera sección, se analiza el plan de la obra y sus filiaciones intelectuales, especialmente con los historiadores del movimiento obrero francés y el abogado e intelectual norteamericano Carrol D. Wright. Especial interés tiene el destacar el uso que hizo Poblete de las memorias de prueba de los estudiantes de Derecho, los que durante la década de 1930 y 1940 ofrecieron los primeros estudios en Chile sobre el movimiento obrero.

En términos metodológicos nos hemos propuesto vincular el libro El movimiento obrero latinoamericano dentro de la mirada global que tuvo Poblete de lo social, las condiciones laborales y la evolución del movimiento obrero, yendo más allá de la descripción detallada de la obra. Para ello es clave entender cómo se articulan sus distintos trabajos con momentos profesionales muy particulares, con ciertas síntesis que ofrece en materia socio-laboral y como insumo intelectual para comprender los problemas sociales contemporáneos. Las publicaciones que de Poblete se han considerado, son, principalmente, sus libros, descartando sus artículos en revistas y notas de prensa, esfuerzo recopilatorio que nadie se ha aventurado a hacer hasta el momento. Complementariamente, se pudo trabajar en los archivos de la OIT que se encuentran en la ciudad de Ginebra, donde se tuvo acceso a la carpeta personal de Moisés Poblete como funcionario internacional, la 
que ofrece información relevante sobre las tareas que le fueron encomendadas entre 1927 y 1937.

\section{Lo social y lo laboral a los ojos de un pensador chileno}

Según los registros del personal de la Organización Internacional del Trabajo (OIT), Moisés Poblete Troncoso nació en Chillán en 1893. Titulado de abogado con una memoria sobre legislación de hijos ilegítimos ${ }^{3}$, desde muy joven mostró una preocupación por los problemas nacionales que afectaban al país, aunque sin involucrarse en la política contingente. En 1920 publicó un ensayo titulado El balance de nuestro seudo régimen parlamentario, criticando el sistema parlamentario chileno y mostrándose dispuesto a avanzar hacia una reforma política que reforzara el poder presidencial ${ }^{4}$.

Una vez electo Arturo Alessandri como Presidente de la República, con una plataforma programática que incluía una serie de reformas sociales, legislación laboral, mayor intervención del Estado en los conflictos laborales y el reforzamiento de la institucionalidad, entre otros aspectos, Moisés Poblete fue nombrado director de la Oficina del Trabajo, institución que había aumentado sus atribuciones, pasando de ser una agencia de estadística a una de inspección laboral ${ }^{5}$. Desde su cargo, Poblete se involucró muchas veces de manera personal en la solución de algunas huelgas, además de dictar conferencias y apoyar de forma entusiasta la reforma legislativa, redactando, por ejemplo, el proyecto de Código Laboral que el Presidente Alessandri presentó al Congreso en junio de 1921.

Durante la primera mitad de la década de 1920 Poblete publicó una recopilación sobre la legislación social chilena junto a Óscar Álvarez Andrews ${ }^{6}$. En ella destacó la importancia de los primeros congresos internacionales promovidos por la OIT y las reuniones panamericanas en la difusión de nuevos principios sociales y laborales. Poblete y Álvarez se mostraron críticos frente al pobre desarrollo de la legislación obrera, responsabilizando al Estado y las doctrinas liberales herederas del economista francés Courcelle-Seneuil por esta situación. Sin embargo, reconocían un cambio en la importancia que las autoridades le estaban dando al problema social en Chile, destacando el papel de las universidades al respecto y la promoción de los cursos de Economía Social.

El interés de Poblete por conectar sus preocupaciones sociales con las instancias internacionales comenzó a aparecer en esta época, lo que explica que se radicaran en la Oficina del Trabajo las relaciones con la OIT, desplazando con ello al Ministerio de Relaciones Exteriores. También se destaca su participación en la Conferencia

\footnotetext{
${ }^{3}$ Moisés Poblete, Legislación sobre hijos ilegítimos (cuestión social), Santiago, Imprenta El Progreso, 1912.

${ }^{4}$ Moisés Poblete, El balance de nuestro seudo régimen parlamentario, Santiago, Talleres de Numen, 1920.

${ }^{5}$ Sobre la creación y funcionamiento de las primeras oficinas del trabajo, tanto en Chile como en el extranjero, ha tenido un gran interés en los últimos años. Véase, por ejemplo, Juan Carlos Yáñez, La intervención social en Chile (1907-1932), Santiago, Ril Editores, 2008; Juan Suriano, "El Departamento Nacional del Trabajo y la política laboral durante el primer gobierno de Hipólito Yrigoyen", en Mariano Ben Plotkin y Eduardo Zimmermann (compiladores), Los saberes del Estado, Buenos Aires, Edhasa, 2012.

${ }^{6}$ Moisés Poblete y Oscar Álvarez, La legislación social obrera chilena (recopilación de leyes y disposiciones vigentes sobre el trabajo y la previsión social, Santiago, Imprenta Santiago, 1924.
} 
Panamericana de 1923 como secretario de la delegación chilena, organizadora del encuentro. En esa calidad fue el responsable, a solicitud del político liberal Manuel Rivas Vicuña, de introducir una resolución para que en las futuras conferencias panamericanas se estudiaran las temáticas sociales ${ }^{7}$. En 1924 participó en el primer Congreso de Economía Social en la ciudad de Buenos Aires, con un estudio sobre la legislación social.

El desarrollo profesional de Moisés Poblete y su pertenencia a una red latinoamericana de intelectuales facilitó su vinculación con la OIT, ayudando a promover, por ejemplo, la revista Informaciones Sociales y apoyando los contactos entre Albert Thomas (director de la OIT) y diferentes personalidades políticas y sindicales de Brasil, Uruguay y Argentina ${ }^{8}$. Los informes que elaboró para la OIT, a propósito del programa del gobierno de Alessandri y los avances en materia de legislación laboral, dan cuenta no solo del cumplimiento de los compromisos asumidos por las autoridades chilenas en materia social, sino también de la cercanía, incluso amistad, que la relación epistolar fue generando entre el funcionario chileno y Thomas.

El arribo de Moisés Poblete a Ginebra como funcionario internacional, resultó del cruce de dos situaciones un poco fortuitas. En principio, el funcionario chileno se sentía incómodo por la situación política nacional y por la parálisis de la administración pública, en particular del recién creado Ministerio del Trabajo, Asistencia y Previsión Social. Su carrera funcionaria parecía estancada y las posibilidades de crecimiento profesional no eran promisorias frente al aumento de poder de Carlos Ibáñez del Campo, quien había accedido a la Vicepresidencia de la República, por lo que este clima político deteriorado habría motivado a Poblete a solicitar un puesto en la OIT. Enseguida, la partida del único funcionario chileno que pertenecía a la institución de Ginebra, Carlos García Palacios, a la Secretaria de la Sociedad de Naciones, dejó vacante un lugar que era necesario llenar, en lo posible, con otro funcionario sudamericano ${ }^{9}$. Valorado por su formación universitaria, sus responsabilidades administrativas y su cátedra en la Universidad de Chile, Poblete aceptó ser contratado de manera temporal, siendo incorporado a la División de Relaciones, en categoría de miembro de Sección B (con un sueldo de 1000 francos al mes). Sus funciones fueron las siguientes: a) ocuparse de las relaciones con Chile, b) dirigir la preparación de un volumen de la serie legislativa sobre la legislación social del continente, c) intensificar el esfuerzo de ratificación de los países sudamericanos ${ }^{10}$.

Al parecer razones presupuestarias hicieron imposible cumplir con los deseos de Albert Thomas de ofrecerle un puesto de miembro de Sección A, como estaba contemplado desde un comienzo. En todo caso, frente a los ofrecimientos que habría recibido Poblete de ocupar un empleo oficial en Europa o incluso Chile, Thomas consideró conveniente

\footnotetext{
${ }^{7}$ Guillermo Feliú Cruz, Moisés Poblete Troncoso: la bibliografía de las ciencias sociales, Santiago, Biblioteca Nacional, 1970, p. 11.

${ }^{8}$ Los informes de la visita del director de la OIT, Albert Thomas, a Chile, muestran los primeros contactos entre Poblete y los funcionarios de la organización de Ginebra. Al respecto véase Juan Carlos Yáñez, La OIT en América del Sur. El comunismo y los trabajadores chilenos (1922-1932), Santiago, Editorial UAH, 2016.

${ }^{9}$ Fabián Herrera y Yannick Wehrli, "Le Bureau international du Travail et l'Amérique latine durant l'entre-deux-guerres : problèmes et enjeux", en Isabelle Lespinet-Moret y Vincent Viet, L'Organisation internationale du Travail. Origine, développement et avenir, Rennes, PUR, 2011, p. 159.

${ }^{10}$ Véase el expediente personal de Poblete en los archivos de la OIT, Ginebra, "Extrait du rapport du 3 mai 1927, N³3", Archivo Organización Internacional del Trabajo [En adelante AOIT] P 2037, M. Poblete Troncoso.
} 
reglamentar su situación ofreciéndole un puesto permanente en la División de Informaciones y Relaciones ${ }^{11}$. Con ello, Poblete fue nombrado en 1929 funcionario permanente con un ingreso anual de 15.600 francos suizos.

Para Poblete, la incorporación a la OIT le significó acrecentar aún más sus competencias y transformarse rápidamente en un experto regional, debiendo, como una de sus primeras tareas, compilar la legislación social del continente americano. Este trabajo dio origen a un volumen doble titulado Legislación social de América Latina (1928-1929) ${ }^{12}$ con el cual se cumplió la exigencia reglamentaria que tenía la OIT de que al cabo de diez años de aprobados los primeros convenios se debía realizar un estudio sobre el estado de avance en su aplicación. En la época, Moisés Poblete Troncoso era una de las personalidades más importantes en lo que concierne el conocimiento de la legislación social del continente americano, permitiéndole sus antiguas funciones como director de la Oficina del Trabajo tener un manejo directo de la gestión de los organismos de inspección laboral y entablar relaciones con las instituciones similares de otros países, así como con personalidades del mundo intelectual, político y sindical latinoamericano. De esta forma, cuando la OIT se propuso elaborar ese informe de la realidad legislativa de la región no había ningún otro profesional capaz de coordinar un esfuerzo de tal naturaleza. Si bien fue redactado en la ciudad de Ginebra durante 1927, la filiación con sus primeras publicaciones sobre el estado de la legislación social en Chile resulta evidente ${ }^{13}$. Además, este informe debe ser enmarcado en una perspectiva de largo plazo, siendo la base de una publicación del mismo Poblete de 1936, la cual reúne la bibliografía social de los países latinoamericanos, ofreciendo un verdadero catálogo actualizado de obras y autores sobre el tema ${ }^{14}$.

Todos estos trabajos de compilación de la legislación social le permitieron a Poblete dar un salto cualitativo al entablar nuevas relaciones con el medio latinoamericano, pensar la realidad continental desde un plano más global y ayudar en el esfuerzo de coordinación del trabajo legislativo que la OIT proyectaba para la primera década de su creación. En cuanto a nuestro estudio, podemos señalar que estos contactos, construidos en el curso de los años, fueron fundamentales al momento de utilizar esa red de amistades para comenzar a redactar El movimiento obrero latinoamericano.

Una vez instalado en Ginebra, y pese a las restricciones presupuestarias que impuso la crisis económica de 1929, Poblete viajó a Chile en 1930, donde realizó una serie de conferencias y tomó contactos con distintas autoridades, además de redactar informes sobre el gobierno del general Carlos Ibáñez del Campo. Recién en 1934 tuvo la posibilidad de participar en una nueva misión a latinoamericana, en el marco de los estudios y asesoría sobre las instituciones de previsión social y la reorganización del Ministerio del Trabajo de Cuba, donde recibió elogiosos comentarios por su labor. Sin embargo, fue la convocatoria a la Primera Conferencia Interamericana del Trabajo de 1936 la que permitió demostrar sus cualidades organizativas. Esta Conferencia se realizó por la invitación del gobierno de Chile durante el transcurso de la Conferencia Internacional del Trabajo de Ginebra en 1935,

11 Nota de Mario Viple, 16 de mayo de 1929, AOIT, P 2037, M. Poblete Troncoso.

12 Moisés Poblete, La legislación social de América Latina, vol. 1-2, Ginebra, OIT, 1928-1929.

${ }^{13}$ Poblete y Álvarez, op. cit.

${ }^{14}$ Moisés Poblete, Ensayo de bibliografía social de los países hispanoamericanos, Santiago, La Nación, 1936. 
mostrándose interesado el gobierno de Arturo Alessandri (1932-1938) en consolidar sus relaciones con la OIT luego de la muerte de Albert Thomas en 1932. Por otra parte, el rol asignado a Poblete en la organización de esta Conferencia permite entender que con este evento se cerraba un primer ciclo de vinculaciones internacionales iniciado en 1920, con los primeros contactos entre Moisés Poblete, Arturo Alessandri y Albert Thomas, no siendo extraño que estas antiguas vinculaciones expliquen la disposición de la OIT a convocar una primera Conferencia Interamericana del Trabajo en la ciudad de Santiago de Chile. A propósito de esta Conferencia, Poblete publicó una investigación sobre los problemas sociales y económicos del continente, una suerte de balance sobre los progresos sociales de la región y que al mismo tiempo puede ser leído como un escrito realizado por un intelectual de primera línea a fin de llamar la atención sobre las visiones prejuiciosas que la intelectualidad europea y norteamericana tenía sobre el continente, señalando claramente que los avances sociales en latinoamericana eran proporcionalmente superiores a aquellos de los países europeos ${ }^{15}$. De manera explícita destacaba una intelectualidad hispanoamericana preocupada por las cuestiones sociales y laborales, entre los cuales se pueden nombrar a los argentinos Juan Justo, Alfredo Palacios, Alejandro Unsain, Augusto Bunge, Tomás Amadeo, José Ingenieros y al mexicano Vicente Lombardo Toledano ${ }^{16}$.

Las resoluciones de la Conferencia Interamericana del Trabajo de 1936 tuvieron dos consecuencias concretas en las tareas que Poblete realizaba en la institución de Ginebra. En primer lugar, se recomendó al Consejo de Administración de la OIT la realización de diversos estudios sobre las condiciones de trabajo en la agricultura y la situación de la población indígena. En este último caso, se le asignó a Poblete la tarea de llevar a cabo una investigación sobre la condición indígena en el Perú, el cual fue publicado en $1938^{17}$. Uno de los aportes principales de este estudio fue determinar la cantidad de indígenas que habitaban el país y sus modalidades de producción en la agricultura e industria. Además, propuso una serie de medidas legales y educativas, con el fin de evitar las formas de trabajo compulsivo y lo extendido que estaba el uso del alcohol y de la coca entre la población indígena. En segundo lugar, la Conferencia Interamericana del Trabajo fue la instancia para que los delegados expresaran una serie de demandas en orden a acercar aún más las tareas de la OIT al continente, obligando a crear nuevas oficinas de corresponsales, entre ellas una en Santiago de Chile ${ }^{18}$. El responsable de organizar dicha oficina fue Moisés Poblete, el funcionario más calificado de la OIT en materias regionales. Al parecer el traslado a América del Sur, acordado a comienzos de 1937, suponía "proseguir las investigaciones sobre las condiciones de vida y de trabajo de los indígenas en ciertos países y para cumplir otras tareas", como lo confidenció el mismo Poblete en una carta ${ }^{19}$. En ella proponía una estadía de dos años en el cargo de corresponsal en Santiago, pero realizando las mismas funciones en países vecinos. Esta estadía, que se transformó en definitiva, le permitió desde

\footnotetext{
${ }^{15}$ Moisés Poblete, Problemas sociales y económicos de América Latina, Santiago, Universidad de Chile, 1936.

${ }^{16}$ Ibid., p. 2.

${ }^{17}$ Moisés Poblete, Condiciones de vida y de trabajo de la población indígena del Perú, Ginebra, OIT, 1938,

${ }^{18}$ Luego de la Conferencia Interamericana del Trabajo de 1936 se crearon, además de Santiago, las corresponsalías de la Habana, Caracas, Montevideo, Lima y Bogotá. Véase Segunda Conferencia Interamericana del Trabajo, Memoria del Director, Ginebra, OIT, 1939, p. 84.

${ }^{19}$ Carta confidencial de Poblete al director de la OIT, del 1 de febrero de 1937, AOIT, P 2037, M. Poblete Troncoso.
} 
Chile continuar con sus responsabilidades de elaborar informes sobre la región y participar en distintas reuniones internacionales, como la Conferencia Interamericana del Trabajo de la Habana de 1939 y la Conferencia Internacional del Trabajo de Nueva York de $1941^{20}$.

A su regreso al país se integró a una red de intelectuales que se había formado al alero de la Universidad de Chile. En 1939 ingresó a la Facultad de Derecho de esta Universidad como profesor extraordinario de Economía Social y Legislación de Trabajo. En la época la Facultad de Derecho comprendía un cuerpo variado de profesores y de programas interesados en las problemáticas sociales y laborales, tendencia que seguía aquella de los años 1910 y 1920. Figuras destacadas eran los profesores Francisco Walker Linares, Alfredo Gaete, Héctor Escribar y Carlos Vergara, quienes en la década de 1930 y 1940 promovieron los primeros estudios sobre el movimiento obrero chileno y perspectivas comparadas entre la legislación laboral chilena y la del resto del continente. La presencia de este perfil de profesores con importantes vínculos internacionales, incluso con el trasfondo de la Segunda Guerra mundial, y el apoyo de la misma Universidad de Chile, fue favorable para el trabajo de Poblete. En 1942 tuvo una destacada participación en la organización de la Primera Conferencia de Seguridad Social realizada en Santiago, publicando para la ocasión un nuevo balance sobre la legislación social del continente americano, el cual incluyó algunos artículos ya publicados y conferencias dictadas entre 1939 y $1940^{21}$.

Es necesario precisar que Poblete compartía con otros intelectuales de su generación, formados en las instancias internacionales, la creencia de que la guerra traería un balance positivo a la hora de juzgar los avances del continente en materia social. Así como la Primera Guerra Mundial había significado la toma de conciencia de la necesidad de promover la paz y la justicia social como valores universales, la Segunda Guerra Mundial señalaba Poblete- debía promover una nueva sociedad sustentada en la seguridad social y económica.

Para la región, que se había mantenido alejada de la guerra y donde se habían desarrollado diversas reuniones internacionales, se habrían perspectivas auspiciosas:

No creemos, sin embargo, que la guerra actual traiga una transformación total en las actuales concepciones y realizaciones económico-sociales de los países hispanoamericanos. Pensamos, eso sí, que la evolución económico-social de América va a acelerarse, y que va a intensificarse, en algunos países y a cimentarse y perfeccionarse en otros, las conquistas sociales $^{22}$.

Según Poblete, frente a un mundo convulsionado como el que ofrecía la Segunda Guerra Mundial había que proponer un programa de cambios sujeto a la planificación y a la evaluación de los expertos.

Un dato ilustrativo dice relación con la posición político-ideológica de Moisés Poblete. Si bien no se le conoce militancia formal en ningún partido, colaboró con la

\footnotetext{
${ }^{20} \mathrm{Su}$ experiencia en esa conferencia es recogida en Moisés Poblete, La Conferencia internacional del trabajo de Nueva York: 27 de octubre-6 de noviembre de 1941, Santiago, Imprenta Universitaria, 1942.

${ }^{21}$ Moisés Poblete, Evolución del Derecho Social en América, Santiago, Editorial Nascimiento, 1942.

${ }^{22}$ Ibid., p. 16.
} 
administración de Arturo Alessandri (1920-1925) y siempre tuvo una gran consideración por su obra de gobierno. De tendencia liberal, puede ser considerado un social-demócrata, apoyando durante su vida un proyecto de cambio social de carácter evolutivo y asignándole al movimiento obrero la labor de educar al pueblo, más bien con un perfil técnico y en asociación con los organismos públicos y organizaciones internacionales como la OIT. A diferencia de muchos de su generación, nunca coqueteó con el corporativismo, ideología a la cual le asignaba poca densidad teórica y la situaba cercana al autoritarismo. Por otra parte, si bien no fue un anti-comunista, y reconoció al Partido Comunista de Chile como una organización democrática y partícipe del sistema, fue crítico de lo que llamaba "sindicalismo revolucionario", con inclinaciones internacionalistas y permeado por los intereses políticos ${ }^{23}$.

\section{América Latina: Hacia la unidad sindical}

Los esfuerzos del movimiento obrero por la unidad latinoamericana datan de comienzos del siglo XX, cuando en 1918 fue creada la Confederación Obrera Panamericana (COPA), primera organización sindical de carácter continental, con el apoyo de la Federación Americana del Trabajo (FAT). Sin embargo, y a pesar de su importancia simbólica, no pudo superar dos problemas concernientes a su fundación. Primero, respondía a las tentativas de los Estados Unidos de controlar el movimiento obrero latinoamericano, apoyándose en las posiciones reformistas de algunos sectores obreros frente al avance del comunismo y, segundo, al seguir los lineamientos de la FAT, su influencia se vio reducida a América del Norte ${ }^{24}$.

De manera paralela, la Federación Sindical Internacional (FSI) inició una política de acercamiento a las organizaciones obreras latino-americanas, en el contexto de los esfuerzos del socialismo europeo por abrir las puertas de la II internacional a las vanguardias socialistas latinoamericanas ${ }^{25}$.

Finalmente, la Internacional Sindical Roja (ISR) también buscó unificar el movimiento obrero del continente. A fines de 1927 los delegados sindicales de América Latina, invitados a la celebración del décimo aniversario de la Revolución Rusa, tuvieron en Moscú la Primera Conferencia Sindical Latinoamericana, que reunió a representantes de Argentina, Brasil, Colombia, Ecuador, México, Uruguay y Chile ${ }^{26}$. Las resoluciones de la Confederación Sindical Latinoamericana (CSLA) se orientaron hacia la promoción de la unidad sindical del continente frente al imperialismo de los Estados Unidos, el apoyo al principio de la lucha de clases, el rechazo al sindicalismo reformista de la COPA y la formación de un amplio frente de trabajadores y de campesinos, entre otras resoluciones ${ }^{27}$. Desde la creación de la CSLA hasta su primer congreso en Montevideo en 1930, los

\footnotetext{
${ }^{23}$ Moisés Poblete, La organización sindical en Chile y otros estudios sociales, Imprenta Ramón Brías, Santiago, 1926, p. 13.

24 Ricardo Melgar, El movimiento obrero latinoamericano, Alianza Editorial, México, 1988, pp. 243-258.

${ }^{25}$ Ibid., p. 253. Además véase Amaro Del Rosal, Los congresos obreros internacionales en el siglo XX. De 1900 a 1950 , Grijalbo, México, 1963, p. 384.

26 Melgar, op. cit., p. 264.

${ }^{27}$ Ibid., p. 265.
} 
sindicatos comunistas promovieron huelgas de carácter insurreccional y la formación de soviets locales y regionales ${ }^{28}$. La línea política de la CSLA, coherente con los postulados de la ISR y de la III Internacional, se impuso como objetivo principal la revolución continental, rechazando toda alianza con los sectores reformistas, no solamente de la burguesía latinoamericana, sino también del movimiento obrero ${ }^{29}$.

Una razón que explica las condiciones favorables para la unidad sindical de la región es el cambio de la política de la Internacional Comunista a partir de su VII Congreso (1935), pasando de una estrategia llamada "clase contra clase" a la formación de "frentes populares", que consistió en una táctica de frente único antifascista, lo que supuso la movilización de masas y la alianza política con los sectores de la burguesía nacional. Luego de este cambio, la política de la CSLA favoreció la lucha por la seguridad social, el aumento de salarios, la defensa de las conquistas ganadas por el movimiento obrero y la oposición a la guerra imperialista. A pesar de esto, y en nombre de la creación de frentes populares, la CSLA fue disuelta en 1936 de acuerdo a las resoluciones de la III Internacional.

Estos esfuerzos de unificación del movimiento obrero continental se aceleraron a partir de septiembre de 1938, con la realización de un Congreso de los Sindicatos Obreros de América Latina, bajo el apoyo de la Confederación de Trabajadores de México (CTM). Los principales representantes sindicales del continente participaron en este encuentro. El congreso acordó constituir la Confederación de Trabajadores de América Latina (CTAL) e instalar su sede central en México ${ }^{30}$. Como objetivos principales la CTAL se planteó el progreso de la legislación laboral y la colaboración con otras organizaciones de carácter internacional, en especial la Organización Internacional del Trabajo (OIT), adoptando la siguiente resolución: "A los efectos de conseguir la ampliación y la aplicación de la legislación del trabajo en los países de América latina la Confederación de Trabajadores Latinoamericanos colaborará activamente con la Organización Internacional del Trabajo. Al mismo tiempo tratará por todos los medios a su alcance que a las Conferencias anuales concurran delegaciones completas y principalmente delegados de las entidades gremiales más representativas, genuinas y auténticas" $"$.

El personaje más destacado de esta nueva organización continental fue el abogado y dirigente sindical mexicano Vicente Lombardo Toledano, quien la dirigió durante casi treinta años hasta su disolución en 1963. Toledano, marxista con una larga historia de lucha

\footnotetext{
28 Según Ricardo Melgar, la mayoría de estas acciones estuvieron dirigidas contra los intereses de las empresas norteamericanas, ibid.., p. 270.

29 Sin embargo, dos casos extremos marcaron tristemente el fracaso de la revolución continental. En primer lugar, en El Salvador, Agustín Farabundo Martí, dirigente del Partido Comunista, encabezó una revuelta para derrocar la dictadura del General Maximiliano Hernández, revuelta sometida a una represión sangrienta en 1932. En segundo lugar, la Unión Soviética apoyó en 1935 la rebelión de Luis Carlos Prestes y de la Alianza Nacional de Liberación del Brasil (ANL) en contra del gobierno de Getulio Vargas. Su derrota significó la ilegalidad de la ANL y la represión de los comunistas, lo que reforzó el gobierno de Vargas hasta el golpe de Estado de 1945. Véase, Julio Godio, Historia del Movimiento obrero latinoamericano, vol. 3, Editorial Nueva Sociedad, Caracas, 1985, pp. 20-23.

30 Patricio Herrera, "Por 'un único y potente puño proletario' para América Latina: antecedentes continentales e internacionales, 1936-1938”, Cuadernos de Historia, N³9, Santiago, 2013.

${ }^{31}$ Segunda Conferencia Interamericana del Trabajo, Informe acerca de las medidas tomadas para dar cumplimiento a las resoluciones adoptadas por la Conferencia de Santiago de Chile, OIT, Ginebra, 1939, p. 210.
} 
sindical, jugó un papel fundamental en el posicionamiento de la CTAL a nivel continental y en la profundización de sus relaciones con la OIT $^{32}$. Esto le permitió ser el primer representante obrero de origen latinoamericano en formar parte del Consejo de Administración de la organización de Ginebra. Así, los esfuerzos en promover la unidad del movimiento obrero y la formulación de una política de alianza de clases dieron inicio a una nueva etapa en la consolidación de las relaciones entre el movimiento sindical continental y la OIT.

De acuerdo a lo anterior, y si tomamos en serio los esfuerzos de unificación del movimiento obrero antes descritos, se puede considerar que una vez finalizada la Segunda Guerra Mundial estaban dadas las condiciones para reflexionar, desde las ciencias sociales, sobre la evolución del movimiento obrero latinoamericano, ya no solo como un agregado de sindicalismos nacionales, sino que en perspectiva regional, en especial estudiando los proyectos de construcción de una organización sindical continental. Por ello no puede sorprender que sea un abogado, con inclinaciones sociales, y no precisamente un historiador, el que haya escrito la primera historia del movimiento obrero latinoamericano.

El interés de Moisés Poblete por el sindicalismo data de su etapa como director de la Oficina del Trabajo, cuando publicó una de sus investigaciones pioneras: La organización sindical en Chile y otros estudios sociales ${ }^{33}$. Para Poblete la evolución del movimiento de asociación está ligada al avance de la sociedad de derechos, que protege las formas de organización sindical con finalidades de mejoramiento económico, social y cultural de sus miembros. Dicho de otro modo, estudiar la historia del movimiento obrero supone estudiar las leyes que ayudan a mejorar las condiciones laborales, que facilitan su organización y que permiten regular los conflictos entre el capital y el trabajo. En su libro de 1926 dividió las organizaciones de trabajadores en seis tipos:

1.- Las asociaciones mutualistas, de larga data y tendiente a ofrecer programas de asistencia social a partir de la organización de sus propios miembros.

2.- Organizaciones sindicales revolucionarias, contrarias al orden social y de afiliación marxista.

3.- Sindicatos anarquistas, ligados tanto al sindicalismo puro como al anarquismo propiamente tal.

4.- Organizaciones obreras inspiradas en la ideología corporativista y orgánica de la sociedad, asociadas al catolicismo social y a los sectores que proponen el evolucionismo en la construcción de un nuevo orden social.

5.- Organizaciones sindicales de empleados, que comprendían empleados particulares y públicos.

6.- Organizaciones profesionales de los intelectuales, las cuales pueden subdividirse en aquellas que tienen tendencias revolucionarias y las que no las guían un propósito de lucha social. A las anteriores había que agregar las asociaciones patronales.

\footnotetext{
32 Patricio Herrera, "Vicente Lombardo Toledano: nexo entre los obreros latinoamericanos y la OIT", Trabajadores, México, $\mathrm{N}^{\circ} 91,2012$.

${ }^{33}$ Poblete, La organización... op. cit.
} 
Sus viajes por el continente americano como funcionario de la OIT, le permitieron conocer la realidad de los distintos países y trabar contacto con personajes y líderes muy variados, contándose entre ellos a presidentes de la república, intelectuales, funcionarios públicos y dirigentes sindicales. Uno de esos contactos importantes corresponde al dirigente sindical mexicano Vicente Lombardo Toledano, del cual obtuvo información de primera mano sobre la realidad laboral del continente. Este aspecto es destacado por la historiadora norteamericana Fanny Simon -a quien se le reconoce haber realizado uno de los primeros estudios sobre Luis Emilio Recabarren- cuando señala el valor de la obra de Poblete al tener acceso a información que la CTAL había reunido sobre el movimiento sindical latinoamericano ${ }^{34}$.

Sus conocimientos sobre la historia sindical provenían de lecturas de historiadores y economistas franceses, como Edouard Dolléans, Georges Lefranc, François Perroux y André Philip, los que influyeron en sus perspectivas evolucionistas y sistémicas del movimiento obrero. La primera perspectiva suponía que el movimiento obrero debía pasar necesariamente por distintas fases de acuerdo a la evolución del capitalismo y del Estado, y la segunda -lo sistémico- decía relación con que el sindicalismo debía propender al mejoramiento de las condiciones laborales en un marco de desarrollo democrático y no revolucionario. En este último punto las lecturas de la obra de Carrol D. Wright, en especial sus estudios sobre la industria en tanto factor de civilización, fueron fundamentales ${ }^{35}$.

\section{El movimiento obrero latinoamericano (1946): el plan de la obra}

A partir de mediados de los años 1940 se inició una nueva etapa en los estudios de Moisés Poblete, orientada a promover un movimiento obrero de raíz socialdemócrata como parte del desafío que tenían las organizaciones de trabajadores frente al escenario que se abría con el fin de la Segunda Guerra Mundial. Es necesario destacar un aspecto interesante: el libro fue redactado en 1945 y publicado en 1946, a inicios de lo que se llamaría la Guerra Fría y dos años antes de la aprobación en Chile de la Ley de Defensa de la Democracia (1948) que proscribió al Partido Comunista, lo que puede explicar la ausencia de una posición anticomunista clara. Por de pronto se muestra favorable a la acción de la Confederación de Trabajadores de Chile (CTCH) creada en 1936, reconociendo en ella un esfuerzo de organización y defensa de los intereses obreros. Su modalidad de organización, en base a federaciones de diferentes ramas industriales, la superación de las divisiones entre socialistas y comunistas y su lucha en contra de la carestía de la vida (finalidad estrictamente sindical), son aspectos que para Poblete la alejarían de la tendencia revolucionaria por él criticada. Además, hay que considerar que desde su creación la CTCH

\footnotetext{
${ }^{34}$ Véase reseña de Fanny Simon al libro El movimiento obrero latinoamericano de Moisés Poblete, publicada en The Hispanic American Historical Review, 26 (4), 1946, p. 550

35 Carrol D. Wright (1840-1909) fue un abogado norteamericano especialista en temáticas socio-laborales, quien postuló la relación intrínseca entre el sistema de producción industrial y la organización sindical, propendiendo a un desarrollo armónico de ambas como base de una política de conciliación entre el capital y el trabajo. Entre sus obras principales se pueden mencionar, por ejemplo, Relation of Political Economy to the Labor Question (1882) y Some Ethical Phases of the Labor Question (1902).
} 
se mantuvo afiliada a un internacionalismo obrero cercano a los organismos de la Sociedad de Naciones y a la CTAL, en un contexto marcado por el consenso logrado en torno a la defensa de la democracia y la lucha en contra del fascismo.

Poblete fue un fuerte partidario de la organización obrera, a la cual le asignó la responsabilidad de mejorar la condición social y económica de los trabajadores. Para él, la madurez del movimiento obrero debía ser evaluada en relación no solamente a su presencia en los grandes centros de producción, sino también por la promoción de un programa de carácter nacional, lo que suponía reconocer una cierta función política a la organización sindical. En una dimensión internacional, las organizaciones obreras continentales deberían ser la extensión natural de las federaciones nacionales, fundamentales en apoyar el trabajo de la OIT $^{36}$. Luego de la Segunda Guerra Mundial la organización sindical debería evolucionar -según Poblete- de una lógica de lucha de clases hacia una lucha por la democracia social y económica. La legislación nacional e internacional ayudaría, en este mismo sentido, a mejorar las condiciones laborales y orientar el movimiento sindical hacia un programa de defensa de los intereses "superiores y más permanentes de la comunidad".

En El movimiento obrero latinoamericano, Poblete manifestó un reconocimiento explícito a la labor del dirigente sindical mexicano Lombardo Toledano, por construir una organización obrera continental unificada y defensora de los principios que sostuvieron la lucha en contra del fascismo: la democracia, la defensa de la soberanía continental, el apoyo a la política americana del "buen vecino" y el desarrollo industrial como vía de progreso económico-social. El mismo Lombardo Toledano desarrolló lazos importantes con la OIT durante las décadas de 1920, 1930 y 1940². Concretamente, a través de la CTAL, fundada en México en el mes de septiembre de 1938, promovió la legislación social, la sindicalización de los campesinos, el mejoramiento de la condición indígena, los seguros sociales, todas temáticas que estaban en plena concordancia con el trabajo realizado por la OIT en el continente ${ }^{38}$.

El interés de Moisés Poblete por estudiar y promover un movimiento sindical socialdemócrata está relacionado con el segundo dominio de preocupaciones que forman parte de sus investigaciones durante los años 1940 y 1950, como es el consumo y los estándares de vida de la población latinoamericana ${ }^{39}$. Este interés en evaluar la seguridad social y económica de la población se enmarca en el dominio que las instituciones internacionales comienzan a tener sobre el tema ${ }^{40}$. Para Poblete el nivel de vida estaba

\footnotetext{
${ }^{36}$ Moisés Poblete, El movimiento obrero latinoamericano, México, FCE, 1946, p. 8.

${ }^{37}$ Para una perspectiva general sobre Lombardo Toledano véase Robert Millon, Mexican marxist: Vicente Lombardo Toledano, University of North Carolina Press, Chapel Hill, 1966.

38 Sobre los lazos de Toledano con la OIT véase Daniela Spenser, "Vicente Lombardo Toledano envuelto en antagonismos internacionales", Izquierdas, Núm. 4, 2009, http://www.izquierdas.cl/revistas/wpcontent/uploads/2011/07/spenser.pdf, (consultada el 30 de abril de 2015).

${ }^{39}$ Moisés Poblete, El Subconsumo en América del Sur, alimentos, vestuario y vivienda, Santiago, Nascimento, 1946, p. 25 .

${ }^{40}$ En la Carta de las Naciones Unidas, firmada en San Francisco el 26 de junio de 1945 se señala la necesidad de promover la cooperación económica y social de las naciones a fin de elevar el nivel de vida de la población, garantizando un trabajo permanente y condiciones de progreso y de desarrollo económico y social, véase Moisés Poblete, Stándar de vida y desarrollo económico-social, Santiago, Editorial Universitaria, 1956, p. 17. La primera institución que se ocupó del estudio de los niveles de vida fue la OIT, a través de las resoluciones de las diferentes conferencias interamericanas del trabajo. Por ejemplo, en 1933 el gobierno de Chile solicitó una comisión de encuesta para estudiar el problema de la
} 
condicionado por cuatro factores: el salario, la cultura, la legislación protectora del trabajo y el costo de la vida ${ }^{41}$. En lo que concierne a los tres primeros factores, el movimiento obrero podía ayudar a mejorar las condiciones de vida y de consumo de los trabajadores y de sus familias, a través de la promoción de una política de defensa salarial, de programas de ahorro y alimentación, así como el apoyo a la legislación social en torno a nuevas temáticas, como el uso del tiempo libre, la formación profesional y la reinserción laboral. Todo esto debía estar acompañado con una acción del Estado en el área de la producción agrícola, el intercambio comercial y la promoción industrial, disminuyendo la inflación y permitiendo un mayor acceso a los bienes fundamentales como los alimentos, vivienda y vestuario.

El libro El movimiento obrero latinoamericano se organiza en seis capítulos, con un total de 290 páginas, los que abordan el origen del movimiento de asociación profesional, el desarrollo del sindicalismo en los distintos países del continente, el sindicalismo panamericano y las organizaciones sindicales en el mundo de post-guerra.

Chile aparece destacado en las páginas de El movimiento obrero latinoamericano, lo cual se explica por los mayores conocimientos que Poblete tenía sobre el sindicalismo de su país y porque éste respondía de mejor manera al modelo evolutivo de tipo europeo y especialmente francés. En términos de lectura, Poblete utilizó diversas memorias de prueba que fueron defendidas por los futuros abogados de la Universidad de Chile. Sobre esto es necesario hacer una mención aparte a la poca importancia que se le han dado a estas memorias como base de lo que serían los posteriores estudios sobre el movimiento obrero durante los años 1950, y que ha sido conocida como la llamada generación marxista ${ }^{42}$ Al menos cuatro memorias que abordaban el movimiento sindical chileno fueron utilizadas por Moisés Poblete. En primer lugar, en términos cronológicos, se encuentra la memoria de Alfredo Bowen, titulada Ensayo sobre el movimiento sindical y el sindicalismo agrícola (1934), donde se hace una extensa reseña sobre los principales acontecimientos que marcaron la lucha sindical en Chile, responsabilizando el autor al Estado y a la clase patronal por las posiciones extremistas asumidas por los trabajadores. En concreto, la falta de protección social y el rechazo de todo tipo de reivindicaciones, incluso las más justas, habrían forzado a los trabajadores a desarrollar acciones cada vez más violentas y asumir posiciones político-partidistas.

En segundo lugar, aparece citada la memoria de Carlos Valdivia, Sinopsis histórica del mutualismo (1940). Lo interesante de este estudio es que, a diferencia de perspectivas posteriores, como la marxista, reconoce en el desarrollo del mutualismo una escuela de formación cívica entre los trabajadores y la conquista, a través de su gestión autónoma, de mecanismos de bienestar social. Además, abre el espectro de lo que consideramos tradicionalmente como sociedades de socorros mutuos, incorporando asociaciones

alimentación, que dio fruto en la publicación de Carlo Dragoni y Etienne Burnet, L'alimentation populaire au Chili, Santiago, Imprenta Universo, 1938.

${ }^{41}$ Poblete, El Subconsumo, op. cit., p. 25.

${ }^{42}$ Los principales representantes de esta corriente historiográfica son Hernán Ramírez Necochea y Julio César Jobet. 
profesionales como la Sociedad Profesores de Instrucción Primaria y la Sociedad Empleados de Comercio, que participaron activamente en la aprobación de la ley de Instrucción Primaria Obligatoria (1920) y la Ley de Empleados Particulares (1924) respectivamente.

En tercer lugar, se hace referencia a la memoria de Aristodemo Escobar, Compendio de la Legislación social y desarrollo del movimiento obrero en Chile (1940). Este autor, que utiliza algunas de las memorias antes citadas, se inclina más por destacar la labor realizada por una primera generación de humanistas -como la llama- y el papel que jugó la prensa obrera en difundir los ideales sociales y ayudar en la organización obrera, destacando tanto la anarquista como la demócrata-socialista. En términos organizativos valora la experiencia de las mancomunales y las primeras huelgas, junto a la Federación Obrera de Chile. Un punto de inflexión habría sido la ley que aprobó los sindicatos legales en 1924, los que no habrían tenido mayor desarrollo en los primeros años por la reticencia de los trabajadores de participar en ellos. A decir de Escobar, sería -paradójicamente - la dictadura de Carlos Ibáñez del Campo (1927-1931) y su política de represión a las "organizaciones libertarias" lo que habría permitido el crecimiento de los sindicatos legales. Para los años 1930 destaca los esfuerzos de unificación de la Confederación de Trabajadores de Chile y el repunte de la organización sindical con la llegada del Frente Popular (1938). También reconoce el aporte de las asociaciones de empleados en dar forma a un movimiento de trabajadores moderno.

Por último, Poblete utilizó la memoria de prueba de Tulio Lagos, Bosquejo histórico del movimiento obrero en Chile (1941). En ella se señala que el desarrollo de un movimiento sindical moderno se había producido a partir de comienzos del siglo XX. Las huelgas, en especial la de Valparaíso en 1903, la de Santiago en 1905 y la de Iquique en 1907, son vistas como resultado de crisis económicas, sin motivaciones político-ideológicas y sin liderazgo sindical. Solo en la década de 1920 -a decir de Lagos- con la incorporación de ideologías de disolución social y el aumento de los conflictos obreros, se habría forzado a los partidos políticos a incorporar en sus programas la "cuestión social". Tulio Lagos formula una crítica a la Federación Obrera de Chile por su adhesión a la Internacional Sindical Roja, lo cual habría provocado una división en las organizaciones obreras y la consiguiente dependencia de partidos políticos. Otro dato no menos significativo es que reconoce la importancia de otros movimientos sociales en la configuración de un movimiento de trabajadores, como la lucha de los arrendatarios y de los empleados públicos, además de algunos intentos de sindicalización campesina.

En síntesis, estas memorias de prueba ofrecían perspectivas innovadoras para pensar el movimiento obrero chileno y latinoamericano, destacando al menos tres aspectos: la validación de la organización sindical como el fruto de un proceso evolutivo de luchas sociales; el pensar en un movimiento de trabajadores que incluye no solo a los sindicatos obreros, sino también organizaciones mutualistas y sindicatos profesionales (empleados); y, por último, el ofrecer una periodización que ha sido validada hasta el día de hoy por la historiografía, que incluye tres etapas. La primera, se inicia a mediados del siglo XIX con la creación de las primeras organizaciones de socorros mutuos. La segunda, que comienza con el despuntar del siglo XX, está asociada al surgimiento de las sociedades de resistencia, mancomunales y primeros sindicatos asociados a las principales industrias, y que tuvo en la 
creación de la Federación Obrera de Chile en 1909 su punto de inflexión. Un tercer periodo es el que comienza en los años 1920 -y que se consolida en los años 1930- en un marco de aprobación de leyes sociales, centralización sindical y definición ideológica ${ }^{43}$.

Las ideas centrales que resumen el pensamiento de Poblete contenido en $E l$ movimiento obrero latinoamericano, pueden organizarse en aquellas que dicen relación con el movimiento de trabajadores a nivel continental y las que hacen referencia al caso chileno en específico. Sobre el primer punto se pueden señalar las siguientes ideas presentes:

1.- El desarrollo del sindicalismo en cada uno de los países latinoamericanos ha debido sortear dificultades de orden político, la presencia de ideologías de ruptura social y el insuficiente desarrollo industrial.

2.- La madurez del movimiento sindical latinoamericano se aprecia no solo por la búsqueda de proteger sus intereses profesionales inmediatos, sino también por integrarse a la vida social y económica del país y trabajar con el Estado en aras del bienestar colectivo.

3.- El movimiento obrero moderno es aquel que tiene un dominio de los problemas sociales y económicos actuales, y que se interesa en participar en las discusiones sobre previsión social, fomento productivo y asociación.

4.- El movimiento obrero de post-guerra (1945 en adelante) debe ser de raíz continental o no será un movimiento obrero capaz de articularse con los bloques mundiales y agencias internacionales.

En cuanto a los principales planteamientos que contiene el libro sobre el movimiento obrero chileno, podemos sintetizarlos en cinco puntos:

1.- Si bien las organizaciones mutualistas fueron experiencias fundamentales en el desarrollo de un primer movimiento de asociación profesional, no habrían sido capaces de recoger las crecientes demandas de los trabajadores y dar cuenta de las nuevas exigencias que presentaba la organización industrial. Sin embargo, para Moisés Poblete su desarrollo no termina con la aparición de los sindicatos, sino que continúa en paralelo con éstos. Prueba de ello es la convocatoria en 1939 a la Convención Nacional Mutualista que reunió a 197 sociedades, adhiriéndose posteriormente 215, en lo que se conocería como Confederación Mutualista ${ }^{44}$.

2.- La aparición de ideologías de crítica social entre los trabajadores supuso el nacimiento de la "conciencia de clase", a lo cual ayudó en un comienzo la prensa como difusora de ideas, inicialmente anarquistas. Si bien se mencionan episodios de lucha social en la zona central del país, asociados a determinados gremios, habría sido en la zona salitrera donde se produjo el origen del movimiento obrero, "con un contenido marxista bien definido, antes que en el resto de América" ${ }^{45}$. Esta tendencia a minimizar el aporte anarquista, por un lado,

\footnotetext{
${ }^{43}$ En este punto no podemos dejar de citar un estudio que puede ser considerado el primer escrito histórico sobre el movimiento obrero chileno, como es el de Luis Galdames, titulado "Los movimientos obreros en Chile", presentado al Primer Congreso Científico Panamericano (1908). Sin embargo, no aparece citado en el libro de Poblete. Para un estudio sobre el escrito de Galdames, véase Juan Carlos Yáñez "Luis Galdames: un escrito fundacional de la historia del movimiento obrero chileno (1907)", Boletín de la Academia Chilena de la Historia, $\mathrm{N}^{\circ} 120$, 2011, pp. 147-174.

${ }^{44}$ Poblete, El movimiento op. cit., p. 123.

${ }^{45}$ Poblete, El movimiento op. cit., p. 126.
} 
y a sobredimensionar la región salitrera como el origen del movimiento obrero, por otro, serán aspectos característicos de la historiografía marxista desde la década de 1950.

c) Si el desarrollo del sindicalismo chileno es más bien tardío con respecto a otros países del continente y europeos, esto se explicaría de acuerdo a Poblete por el retardo del proceso de industrialización, el cual se produce solo a partir de la Primera Guerra Mundial. Por otra parte, la ausencia de población inmigrante, a diferencia de otros países como Argentina, no permitió el flujo de experiencias externas. Por último, la coyuntura de crisis por los efectos de la Primera Guerra Mundial y el ejemplo de la Revolución Rusa tienen como consecuencia el fortalecer el movimiento sindical.

d) La resistencia patronal a la organización de los trabajadores y la represión de las huelgas, fue un factor que ayudó a radicalizar el movimiento de trabajadores, al no encontrar una contraparte dispuesta a mejorar las condiciones laborales en el marco de una naciente legislación social. Poblete recuerda que cuando se presentó el proyecto de legislación social durante la presidencia de Arturo Alessandri recibió una fuerte oposición "en los elementos capitalistas del Congreso Nacional, compuesto en una proporción altísima de propietarios, industriales y personas vinculadas con el comercio" 46 . La fragmentación del movimiento sindical tampoco habría ayudado a centralizar y conducir por los cauces institucionales las demandas gremiales, al menos hasta los años 1930, existiendo diversas centrales obreras, agrupadas tanto por inclinación ideológica como por rama industrial.

e) Durante el gobierno de Ibáñez (1927-1931) hubo una amplia promoción de la organización sindical, apoyada por la ley de sindicatos de 1924, la que buscó ser controlada por la autoridad. No solo los obreros, sino que también los médicos, ingenieros, profesores, abogados y arquitectos buscaron aprovechar las condiciones favorables para la organización, algunos de los cuales tendrán participación activa en la caída de su gobierno. Los gremios que aparecen destacados son los ferroviarios, panaderos, marítimos, empleados, entre otros.

\section{Consideraciones finales}

Moisés Poblete Troncoso puede ser considerado un intelectual de primer orden, tanto a nivel nacional como continental, que hizo aportes en materia legislativa, organización sindical, estándares de vida, consumo, organizaciones internacionales, organismos técnicos del trabajo, tiempo libre, derechos humanos, entre otros. La diversidad de estudios, así como su complejidad y alcances, pueden explicar, en parte, la falta de reconocimiento que la historiografía chilena ha hecho de su obra, lo que se refleja en la escasez de citaciones que se encuentran en los trabajos sobre movimiento obrero, legislación laboral o estándar de vida.

Sin embargo, otra razón puede explicar este desconocimiento de la obra del intelectual chileno. Su filiación profesional con el mundo del derecho, sus enfoques sociológicos e historiográficos, sitúan a Poblete en un campo de las ciencias sociales en formación y

\footnotetext{
${ }^{46}$ Poblete, El movimiento op. cit., p. 141.
} 
difícil de encasillar, lo que dio origen a una obra pionera pero que no tuvo grandes continuadores ni discípulos ${ }^{47}$.

El movimiento obrero latinoamericano no fue una obra aislada dentro del itinerario intelectual de Poblete, sino que se insertó en las antiguas preocupaciones que tuvo sobre la legislación social, las condiciones laborales y la organización de los trabajadores. Puede ser considerada como el fruto de un sólido conocimiento que tuvo, desde sus cargos como director de la Oficina del Trabajo hasta sus actividades como funcionario internacional, sobre el sindicalismo continental y los procesos sociales que se daban en el continente.

En términos de contenido, El movimiento obrero latinoamericano se sitúa en la aparición de los primeros estudios sobre la historia del movimiento obrero, provenientes de las memorias de prueba de los futuros abogados y donde se destacan los orígenes de las organizaciones obreras y su papel en la consolidación de la sociedad industrial y del Estado de Bienestar. En definitiva, tanto los marcos temporales, líneas evolutivas, grupos representativos y las modalidades de expresión que estudió Poblete para caracterizar el movimiento obrero latinoamericano, marcaron una pauta en lo que serían los futuros trabajos historiográficos, hoy considerados como clásicos.

\section{Fuentes de Archivo}

Archivo de la OIT, Expedientes personales

P 2037. Moisés Poblete Troncoso

\section{Referencias bibliográficas}

Del Rosal, Amaro, Los congresos obreros internacionales en el siglo XX. De 1900 a 1950, Grijalbo, México, 1963.

Dragoni, Carlo y Burnet, Etienne, L'alimentation populaire au Chili, Santiago, Imprenta Universo, 1938.

Godio, Julio, Historia del Movimiento obrero latinoamericano, vol. 3, Editorial Nueva Sociedad, Caracas, 1985.

Herrera, Fabián y Wehrli, Yannick, "Le Bureau international du Travail et l'Amérique latine durant l'entre-deux-guerres: problèmes et enjeux", en Isabelle Lespinet-Moret y Vincent Viet, L'Organisation internationale du Travail. Origine, développement et avenir, Rennes, PUR, 2011.

Herrera, Patricio, "Vicente Lombardo Toledano: nexo entre los obreros latinoamericanos y la OIT", Trabajadores, México, N91, 2012.

Herrera, Patricio, "Por 'un único y potente puño proletario' para América Latina: antecedentes continentales e internacionales, 1936-1938”, Cuadernos de Historia, N³9, Santiago, 2013.

${ }^{47}$ Tardíamente en 1972 Guillermo Feliú Cruz le hizo un reconocimiento en el ensayo ya citado Moisés Poblete Troncoso... op.cit. 
Iriye, Akira, Global Community. The Role of International Organizations in the Making of the Contemporary World, Berkeley, California University Press, 2002.

Melgar, Ricardo, El movimiento obrero latinoamericano, Alianza Editorial, México, 1988,

Millon, Robert, Mexican marxist: Vicente Lombardo Toledano, University of North Carolina Press, Chapel Hill, 1966.

Poblete T., Moisés y Álvarez, Oscar, La legislación social obrera chilena (recopilación de leyes y disposiciones vigentes sobre el trabajo y la previsión social, Santiago, Imprenta, 1924.

Poblete T., Moisés. Legislación sobre hijos ilegítimos (cuestión social), Santiago, Imprenta El Progreso, 1912.

El problema de la producción agrícola y la política agraria nacional, Santiago, Imprenta Universitaria, 1919.

El balance de nuestro seudo régimen parlamentario, Santiago, Editorial Numen, 1920.

Los organismos técnicos del trabajo, Santiago, Imprenta Santiago, 1923.

1926.

La organización sindical en Chile y otros estudios sociales, Santiago, Imprenta Ramón Brias,

Instituciones de previsión social, Santiago, Imprenta Recurba, 1927.

La legislación social de América Latina, vol. 1-2, Ginebra, OIT, 1928-1929.

La aplicación de la legislación social en la América Latina, Ginebra, OIT, 1935.

Ensayo de bibliografía social de los países hispanoamericanos, Santiago, La Nación, 1936.

Problemas sociales y económicos de América Latina, Santiago, Universidad de Chile, 1936.

Condiciones de vida y de trabajo de la población indígena del Perú, Ginebra, OIT, 1938.

La Conferencia internacional del trabajo de Nueva York: 27 de octubre-6 de noviembre de 1941, Santiago de Chile, Imprenta Universitaria, 1942.

Evolución del Derecho Social en América, Santiago, Editorial Nascimiento, 1942.

"Orientaciones de la política económica social de la Post-Guerra", Anales de la Facultad de Derecho, vol. X, n³7-40, enero - diciembre 1944, http://www.analesderecho.

uchile.cl/index. php/ACJYS/article/view/4241/4131.

El movimiento obrero latinoamericano, México, FCE, 1946. 
El Subconsumo en América del Sur, alimentos, vestuario y vivienda, Santiago, Editorial Nascimiento, 1946.

El derecho del trabajo y la seguridad social en Chile, Santiago, Editorial Jurídica, 1949.

Stándar de vida y desarrollo económico-social, Santiago, Editorial Universitaria, 1956.

Saunier, Pierre-Yves y Akira Iriye, The Palgrave Dictionary of Transnational History, Nwe York, MacMillan Publisher, 2009.

Simon, Fanny, Reseña del libro El movimiento obrero latinoamericano de Moisés Poblete, en The Hispanic American Historical Review, 26 (4), 1946.

Segunda Conferencia Interamericana del Trabajo, Informe acerca de las medidas tomadas para dar cumplimiento a las resoluciones adoptadas por la Conferencia de Santiago de Chile, OIT, Ginebra, 1939.

Spenser, Daniela, "Vicente Lombardo Toledano envuelto en antagonismos internacionales", Izquierdas, $\mathrm{N}^{\circ} 4$, 2009, http://www.izquierdas.cl/revistas/wp-content/uploads/2011/07/spenser.pdf, (consultada el 30 de abril de 2015).

Suriano, Juan, "El Departamento Nacional del Trabajo y la política laboral durante el primer gobierno de Hipólito Yrigoyen", en Mariano Ben Plotkin y Eduardo Zimmermann (compiladores), Los saberes del Estado, Buenos Aires, Edhasa, 2012.

Yáñez Andrade, Juan Carlos, La intervención social en Chile (1907-1932), Santiago, Ril Editores, 2008.

Yáñez Andrade, Juan Carlos, "Luis Galdames: un escrito fundacional de la historia del movimiento obrero chileno (1907)”, Boletín de la Academia Chilena de la Historia, º120, 2011.

Yáñez Andrade, Juan Carlos, La OIT en América del Sur. El comunismo y los trabajadores chilenos (1922-1932), Santiago, Editorial UAH, 2016. 